\title{
Complications of cervical spine manipulation therapy: 5-year retrospective study in a single-group practice
}

\author{
David G. Malone, M.D., Nevan G. Baldwin, M.D., Frank J. TomeceK, M.D., \\ Christopher M. Boxell, M.D., Steven E. GAede, M.D., \\ Christopher G. Covington, M.D., and Kenyon K. Kugler, M.D.
}

Oklahoma Spine and Brain Institute, Tulsa, Oklahoma; and Texas Tech University, Author: provide city and state

\begin{abstract}
Object. The authors report a series of 22 patients in whom major complications developed after cervical spinal manipulation therapy (CSMT). A second objective was to estimate the regional incidence of these complications and to compare it with the very low incidences reported in the literature.

Methods. During a 5-year period, practioners at a single group neurosurgical practice in Tulsa, Oklahoma, treated 22 patients, who were markedly worse during, or immediately after, CSMT. The details of these cases are reported. The 1995 US Government National Census was used to define the regional referral population for Tulsa. The published data regarding the incidence of serious CSMT-related complications and the rate of CSMTs undertaken nationally were used to estimate the expected number of CSMT-related complications in the authors' region. The number (22 cases) reported in this series was used to estimate the actual regional incidence.

Complications in the series included radiculopathy (21 cases), myelopathy (11 cases), Brown-Séquard syndrome (two cases), and vertebral artery (VA) occlusion (one case). Twenty-one patients underwent surgery. Poor outcomes were observed in three, outcome was unchanged in one, and 17 improved. The number of patients in this series exceeded the expected number for the region.

Conclusions. Cervical spinal manipulation therapy may worsen preexisting cervical disc herniation or cause disc herniation resulting in radiculopathy, myelopathy, or VA compression. In cases of cervical spondylosis, CSMT may also worsen preexisting myelopathy or radiculopathy. Manipulation of the cervical spine may also be associated with higher complication rates than previously reported.
\end{abstract}

KEY WORDS - chiropractic • intervertebral disc herniation • manipulation, orthopedic • neurological deterioration - cerebrovascular disease - vertebral artery

Cervical spinal manipulation therapy is a common modality for the treatment of neck pain and headache with between 18 and 38 million such treatments performed yearly by US chiropractors. ${ }^{46}$ There are several types of spinal manipulation. One type is the high-velocity, lowamplitude thrust technique the goal of which is to provide a "sharp thrust with velocity" to induce a gap in the joint. ${ }^{15}$ Numerous complications have been reported from cervical spinal manipulation (Table 1).2,3,5,7,9,11,12,14,16-18,23,24, 26-29,31,32,36-38,41,42,44,47,49 Some practioners have advocated manipulation of the cervical spine in cases of MR imaging-documented cervical disc herniation. ${ }^{4}$ The use of general anesthesia as an adjunct to cervical spine manipulation in cases of cervical disc herniation has also been

Abbreviations used in this paper: $\mathrm{ACDF}=$ anterior cervical discectomy and fusion; CSMT = cervical spine manipulation therapy; $\mathrm{CT}=$ computerized tomography; $\mathrm{MR}=$ magnetic resonance; $\mathrm{VA}=$ vertebral artery. supported by some authors. ${ }^{20}$ Serious CSMT-related complications are reported to be rare. ${ }^{9,10,18,21,24,35}$ In other studies investiagors have found that these complications occur more frequently. ${ }^{27,28}$ The purpose of this study was to report the types of complications documented in a single group practice of six neurosurgeons.

\section{CLINICAL MATERIAL AND METHODS}

We retrospectively reviewed data obtained in patients who underwent CSMT between January 1, 1993, and January 1,1998 , and were treated by the physicians of a single-group practice. In all cases the following International Classification of Diseases-9 diagnosis codes were established: 722, 722.2, 722.4, 722.71, 723.4. All patients treated by manual practitioners or who underwent spinal manipulation were further evaluated. The following treatment-related results were determined: adverse effect, reaction, or incident. An adverse effect was defined as any 
TABLE 1

Reported complications of CSMT

vascular injury and stroke syndromes
vascular injury
hearing loss
locked-in syndrome
vertebrobasilar distribution infarction
thalamic infarction
dissecting aneurysm of the VA
dissecting hematoma of the internal carotid artery
visual field loss
structural lesions
intradural herniated cervical disc
lumbar disc rupture
rupture of intervertebral disc
odontoid fracture
atlantoaxial injury
neural injury
cerebellar \& brainstem trauma
diaphragmatic paralysis
cauda equina syndrome
quadriplegia (due to CSMT in cases of spinal cord astrocytoma)
myelopathy
radiculopathy
other injury
tracheal rupture (posttracheotomy)
cervical epidural hematoma

detrimental result of the treatment, a reaction as a slight or clinically insignificant short-lived symptom, and an incident as an unexpected event resulting in serious impairment, injury, or fatality. ${ }^{51} \mathrm{An}$ incident could also be termed an irreversible complication. ${ }^{15}$ "Incident"-related criteria included markedly increased pain, numbness, weakness, markedly worsening myelopathy, or vertebobasilar insufficiency symptoms following manipulation. In patients in whom incidents occurred, the preoperative radiographic data, intraoperative findings, and postoperative results were evaluated. These cases were also evaluated for any published contraindication to spinal manipulation therapy.

The data recorded between January 1, 1993, and June 30, 1996, were evaluated further to compare the total number of patients with cervical disc herniation-induced myelopathy and those in whom CSMT was a complicating factor.

\section{Case 1}

This 38-year-old man had complained of neck pain and had undergone two osteopath-administered sessions of cervical manipulation. On the second manipulation, he experienced forced flexion, a sudden increase in pain, new pain radiating down his left arm with numbness in his fingers, and he developed weakness in his left hand. He was seen in consultation by a neurosurgeon and conservative measures were tried, but he did not improve. He underwent MR imaging, which revealed a large C5-6 disc herniation on the spinal cord and left C-6 nerve root causing compression. He underwent a C5-6 ACDF. Intraoperatively numerous large free fragments of disc were found. Postoperatively the patient made a complete recovery.

\section{Case 2}

This 45-year-old man suffered neck and left shoulder pain. He saw a chiropractor who performed neck manipulation. After manipulation, the patient experienced rapid onset of severe arm pain, with numbness in the thumb and index finger of the left hand, and left arm weakness. Radiography revealed a C6-7 disc herniation, a C5-6 disc bulge, and $\mathrm{C} 4-5$ spondylosis and disc herniation. He underwent C-5 and C- 6 corpectomies and iliac crest strut graft- and plate fixation-assisted fusion. Intraoperative findings were spondylosis and a large free disc fragment. Postoperatively, the patient did well. Normal strength and no neck pain were observed at the 3-year follow-up examination.

\section{Case 3}

This 41-year-old woman reported mild neck pain that worsened over time, and she had begun to experience upper-extremity numbness and tingling. She saw a chiropractor who performed neck manipulations. After manipulation, she developed significant pain and diminished strength in her arms. Physical examination demonstrated myelopathy. An MR image revealed a very large central C4-5 disc herniation compressing the spinal cord as well as C5-6 spondylosis. She underwent $\mathrm{C} 4-5$ and C5-6 ACDF. Postoperatively, her arm and myelopathic symptoms resolved, but she continued to complain of chronic neck pain.

\section{Case 4}

This 35-year-old woman initially saw an osteopathic physician for low-back pain. During the course of her treatment, she underwent a mid- and low-region lumbar spinal manipulation. During manipulation, she noted the onset of neck and right shoulder pain. She then saw a chiropractor who performed a series of 10 CSMTs. During these manipulations, right upper- and lower-extremity weaknesses developed. She worked as a dental hygienist and lost fine motor control in her right hand. Physcal examination revealed a Brown-Séquard syndrome. Magnetic resonance imaging demonstrated a very large C5-6 disc that had herniated toward the right side, deflecting the spinal cord to the left side and rotating it (Fig. 1). A C5-6 anterior cervical discectomy was performed and by 3 months her partial Brown-Séquard syndrome had resolved. She had no neck pain. Muscle strength was $5 / 5$ in the deltoids, biceps, triceps, wrist flexors, wrist extensors, and intrinsic. She was able to return to work and perform her job well.

\section{Case 5}

This 48-year-old woman developed neck and left arm pain. She made two visits to a chiropractor who performed neck manipulation on each visit. After the second manipulation, she complained of severe arm pain and left C-6 distribution numbness. Physical examination after manipulation showed left biceps strength of $4 / 5$, brachioradialis, and $4+/ 5$ in the left triceps. She had hyperreflexia with positive Hoffmann signs. Magnetic resonance imaging revealed a large, slightly left-sided C5-6 disc herniation that compressed the cervical cord and nerve roots. She underwent C5-6 ACDF. 


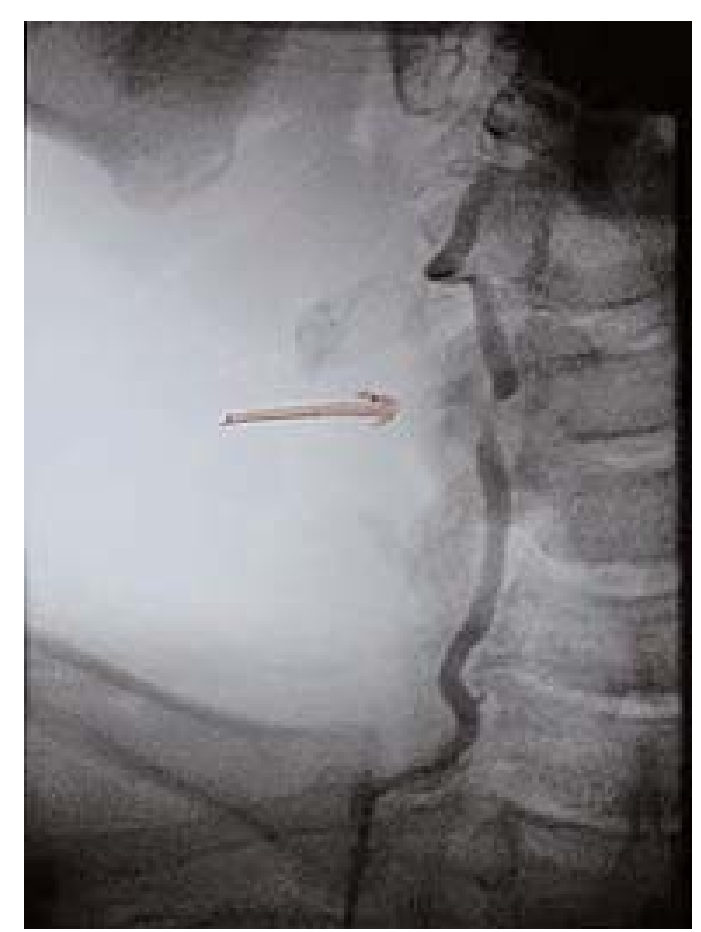

Fig. 1. Case 4. Large cervical disc herniation compressing the spinal cord at C5-6.

\section{Case 6}

This 59-year-old man had a history of chronic neck pain, which developed after undergoing C6-7 allograftassisted ACDF for right-sided C-7 radiculopathy, which was successfully resolved. He sought chiropractic care for his chronic neck pain and underwent a series of neck manipulations. After a series of these sessions, he began to lose function of his hands; the treatments continued, and he began to lose his ability to ambulate. He was then referred to an "arthritis doctor" for treatment of his neck, back, and lower-extremity pain. He eventually sought the care of a neurologist who ordered MR imaging of the cervical spine. Physical examination showed bilateral $4+/ 5$ weakness in the wrist extensors and flexors and intrinsic muscles of the hands at 3/5. Gait was broad based and spastic. Proprioception was diminished in the lower extremities. Magnetic resonance imaging revealed a large C5-6 disc herniation causing marked spinal cord compression and abnormal signal in the cord. Plain radiography demonstrated a C6-7 pseudarthrosis. The patient underwent a C-6 corpectomy and allograft strut- and plate-assisted fusion. At his 6-month follow-up visit he had no neck pain, his gait had markedly improved, and his hand function had improved but he still had intrinsic muscle weakness and difficulty with fine motor control of his hands.

\section{Case 7}

This 38-year-old man suffered right shoulder and neck pain. He underwent one CSMT session and shortly thereafter developed right arm pain, hand numbness, and arm weakness. Physical examination showed weakness in the right biceps, and brachioradialis at $4 / 5$, right triceps at $3 / 5$, with crossed adductor reflexes. Magnetic resonance imaging revealed C6-7 herniated nucleus pulposis compressing the cord, as well as C5-6 spondylosis. The patient underwent a C-6 corpectomy, placement of an iliac crest strut graft, and plate fixation. Intraoperative findings included a large free disc fragment at C6-7. Postoperatively the patient did extremely well with complete resolution of symptoms.

\section{Case 8}

This 44-year-old man developed neck and shoulder pain with numbness in the fingers of his left hand. $\mathrm{He}$ underwent two chiropractic CSMT sessions, which greatly worsened his symptoms and caused new onset of left arm pain. Physical examination demonstrated left thumb numbness and weakness $(+4 / 5)$ of his biceps and triceps. Computerized tomography myelography revealed severe degenerative spondylosis at C5-6 to the left of the midline, as well as C6-7 deformity of the nerve root sleeve on the left. The patient underwent C5-7 iliac crest-assisted ACDF. Intraoperative findings included osteophytic compression of the nerve roots and thecal sac but no disc herniation. Postoperatively the patient had complete relief of symptoms.

\section{Case 9}

This 68-year-old woman had been undergoing follow up for neck and intermittent arm pain. She underwent a series of chiropractic neck manipulations and after one treatment experienced greatly worsened pain with "electric shocks" radiating into her chest and arms. Cervical CT myelography demonstrated disease at $\mathrm{C} 4-5$ in the left facet joint, C5-6 with posterior osteophyte impinging on the theca and nerve roots, and at C6-7 with posterior osteophyte formation compressing the spinal cord. The patient underwent $\mathrm{C} 4-7$ iliac crest- and plate-assisted ACDF. Intraoperative findings included osteophytic disease compressing the spinal cord and nerve roots at each level. Postoperatively the patient improved and has experienced no recurrent arm pain.

\section{Case 10}

This 45-year-old woman was injured in a motor vehicle accident. She had initial complaints of neck pain and headache. She underwent chiropractic neck manipulation and developed new symptoms of shocklike pain radiating into her arms. Physical examination showed generally normal status; MR imaging of the cervical spine revealed spondylosis and foraminal narrowing with osteophytic compression of the thecal sac. Conventional conservative therapy failed, and the patient underwent a C5-6 iliac crest-assisted ACDF. Intraoperative findings were osteophytic spurs compressing the thecal sac and nerve roots. At 6 months postoperatively she had no arm or neck pain but continued to complain of headache.

\section{Case 11}

This 43-year-old woman was injured on the job 3 years prior to presentation. During the course of conservative therapy, she sought treatment from a chiropractor. Prior to 
this, she complained of neck and shoulder pain. After CSMT, her neck and shoulder pain greatly increased, and she developed left arm and left leg pain, with loss of upper-extremity strength. Physical examination revealed left wrist extensor weakness of $4 / 5$ and global hyperreflexia. Magnetic resonance imaging revealed a large disc herniation eccentric to the left side at C5-6 with spinal cord compression. She underwent iliac-assisted ACDF. Intraoperative findings were osteophytic spurs and disc herniation with free fragment. At her 6-month follow-up visit she had no residual pain and her strength had returned to normal.

\section{Case 12}

This 53-year-old man had a 2-month history of severe right shoulder and arm pain, as well as right arm numbness and weakness. For treatment, he had gone to a chiropractor who performed manipulation. On the third visit, he heard a popping in his neck and felt a dramatic increase in pain. On physical examination the triceps strength on the right was 4/5. Sensory examination revealed decreased pin-prick sensation in the right C-7 distribution. On MR images, we observed mild cervical spondylosis at C3-4 and C5-6, a left-sided C5-6 disc bulge, and a large C6-7 disc herniation with complete amputation to the rightsided nerve root; mild lateral cord compression was also noted. The patient underwent C6-7 iliac crest-assisted ACDF. Intraoperatively large free disc fragments were found. Three months postoperatively the patient was doing well; normal strength had returned and no neck or arm pain existed.

\section{Case 13}

This 57-year-old man initially suffered neck and shoulder pain. He underwent five chiropractic CSMT sessions. He stated that after the manipulation he experienced significant increase in pain and numbness in his hands. Finally after a few treatments in which this phenomenon continued, his chiropractor ordered radiographic evaluation and recommended that the patient see a neurosurgeon. Physical examination was significant for left biceps and triceps weakness $(+4 / 5)$, wrist flexors, and extensors weakness $(+4 / 5)$, with weakness in the intrinsic muscles of both hands at (3/5), and his gait was mildly spastic. Postmyelography CT scanning revealed a complete myelographic block at C5-6 and a severe hypertrophic spur formation compressing the C5-6 and C6-7 segments. The patient underwent a C-6 corpectomy, placement of an iliac crest bone graft, and plate-assisted fixation. Large free disc fragments with osteophytic compression were found intraoperatively. At 4 months postoperatively, the patient improved but experienced residual burning in both hands, and despite improvement in hand strength he continued to suffer significant hand weakness.

\section{Case 14}

This 39-year-old woman had a long history of headache and was receiving CSMT from an osteopathic physician. Within an hour of cervical manipulation, she began having pain that radiated in her neck down into her shoulder as well as right arm numbness. This was followed by pain radiating down the posterior part of the right leg. Numb- ness persisted in her fingers, and her arm became weak. An MR imaging study revealed a large right-side C6-7 disc herniation, which was confirmed on cervical CT myelography. She continued to experience pain, but her strength improved slightly, and she refused operative therapy. Six months postinjury she continued to suffer some neck pain, but no arm pain, and she continues with conservative treatment.

\section{Case 15}

This 61-year-old diabetic man fell while fishing and suffered neck and back pain. He sought chiropractic treatment and underwent LSMT in one session. Thereafter, he noted greatly increased pain, and bilateral hand numbness developed the day after his neck manipulation. Gait difficulty occurred within days of CSMT, and the patient had to ambulate using a cane. Physical examination showed a broad spastic gait and upper-extremity weakness; left triceps, wrist flexors, and wrist extensors were grade $4 / 5$ whereas right triceps, wrist extensors and wrist flexors were grade $4+/ 5$. No Hoffmann or Babinski signs were present. Magnetic resonance imaging revealed a large herniated C4-5 disc compressing the spinal cord. The patient underwent a C4-5 discectomy and allograft-assisted fusion. Intraoperative findings included a large free fragment of disc material compressing the spinal cord. Postoperatively his strength normalized, his gait improved, but myelopathy remained. This patient had undergone CSMT outside the geographical region of Tulsa.

\section{Case 16}

This 31-year-old woman injured herself at work. She suffered low-back pain and neck pain. She saw a chiropractor and received 21 low-back and neck treatments. Her low-back pain improved, but with each treatment her neck pain worsened and she developed bilateral hand pain and numbness, worse on the right than the left side. Additionally, due to the cervical manipulations, she developed progressive right arm weakness with loss of hand coordination bilaterally. Physical examination showed deltoids strength of $4+15$ bilaterally, biceps of $4 / 5$ bilaterally, triceps of $5 / 5$, and intrinsics of $4+/ 5$ with diminished sensation bilaterally. A Hoffmann sign was present but otherwise her deep tendon reflexes were normal. The MR imaging study revealed a large herniated C5-6 disc, spinal cord compression, and extruded disc fragments. She underwent a C5-6 iliac crest-assisted ACDF. Intraoperative findings included a large free fragment of disc compressing the spinal cord. Postoperatively, she was initially better: her strength returned and she experienced less pain. She began, however, to complain of neck pain, recurrent left arm pain, and a repeated CT myelography scan revealed a pseudarthrosis at the operative site and slight compression of the left C-6 nerve root. She underwent repeated fusion via an anterior approach with placement of an iliac crest graft and plate fixation. After the second surgery she has continued to complain of neck pain and numbness.

\section{Case 17}

This 49-year-old man fell at work and developed hand tingling and neck pain. He underwent CSMT, which re- 
sulted in worsening symptoms of right arm pain and weakness. He had a history of C4-5 fusion. On examination he had diminished grip strength, +3 reflexes, and a positive Hoffmann sign bilaterally. Radiography revealed C3-4 osteophytes, C4-5 fusion, and a large disc herniation compressing the cord at C5-6. A different physician group performed surgery, and this patient's outcome is unknown. His CSMT had been performed outside of the geographical region of Tulsa.

\section{Case 18}

This 43-year-old woman was injured in a motor vehicle accident many years ago and suffered a neck injury of an unspecified type, which responded to chiropractic therapy. Recently her neck pain increased and she underwent CSMT by her chiropractor. This caused increased neck pain and new left arm pain with weakness and numbness. Physical examination showed left triceps weakness and left wrist extensor muscle weakness. There were no signs of myelopathy. Radiographically she harbored a central disc herniation at C5-6 and a large C6-7 disc herniation eccentric to the left. She underwent a two-level iliac crestassisted ACDF. She exhibited complete return of strength and good resolution of her neck pain.

\section{Case 19}

This 52-year-old man had been making numerous visits to his osteopathic physician who performed neck manipulations for chronic neck pain. On his last visit he suffered new onset of left arm and shoulder pain with left arm numbness. Physical examination revealed biceps weakness, absent biceps reflex, and numbness in the C-6 dermatome. Radiographically, a lateral disc rupture was documented at C5-6. He underwent iliac crest-assisted ACDF. His strength improved but he continued to experience neck and shoulder pain, pseudarthrosis was found, repeated instrumentation-assisted fusion was performed, and his symptoms resolved.

\section{Case 20}

This 51-year-old man injured his neck diving into a pool. He developed chronic neck pain and underwent chiropractic neck manipulation. Severe neck, right arm, and shoulder pain developed after one session of manipulation. Physical examination demonstrated right biceps and tricep weakness as well as brachioradialis. Reflexes were grade +3 , and a Hoffmann sign was present bilaterally. Radiography revealed a large bone spur causing cord compression and C5-6 and C6-7 deformation. He underwent C-6 corpectomy, with good result and only slight residual shoulder pain.

\section{Case 21}

This 55-year-old man developed neck pain. He sought chiropractic treatment and underwent 10 CSMTs. He developed gait problems, could not control his right arm or leg, and suffered increased neck pain. During the manipulation maneuvers he noted electrical shooting sensations into his arms and legs. Physical examination demonstrated partial Brown-Séquard syndrome, right-sided weakness, and hyperreflexia. Myelography revealed multilevel cervical spondylosis, with an extremely large disc herniation compressing the cord at $\mathrm{C} 4-5$. He underwent C4-5 iliac crest-assisted ACDF, and exhibited return of his gait function, coordination, and strength to almost normal levels.

\section{Case 22}

This 58-year-old man suffered right shoulder pain and neck pain. On physical examination, mild weakness in the right biceps was noted. After seeking neurosurgical consultation, he sought chiropractic care involving a series of cervical manipulation. After his fifth CSMT, he developed dizziness and brief transient periods of unconsciousness associated with turning his head to the right and extending his neck. He underwent MR angiography, and both VAs were normal. He continued to experience dizziness and transient blackouts associated with turning his neck to the right. He underwent angiography while turning his neck. During the procedure, his right VA became occluded as he turned his neck to the right, and his symptoms recurred (Fig. 2). He underwent VA decompression via an anterior Verbiest approach and an extreme-far lateral disc rupture was found exiting the uncovertebral joint at C3-4, where it impingedon the VA. Postoperatively he experienced complete resolution of his transient ischemic attack symptoms following the far-lateral discectomy.

\section{RESULTS}

The search criteria were fulfilled in 1712 cases seen during the time of the study. Of these, 187 patients saw manual practitioners and 172 received one or more treatments of cervical spinal manipulation. Of the 172 patients

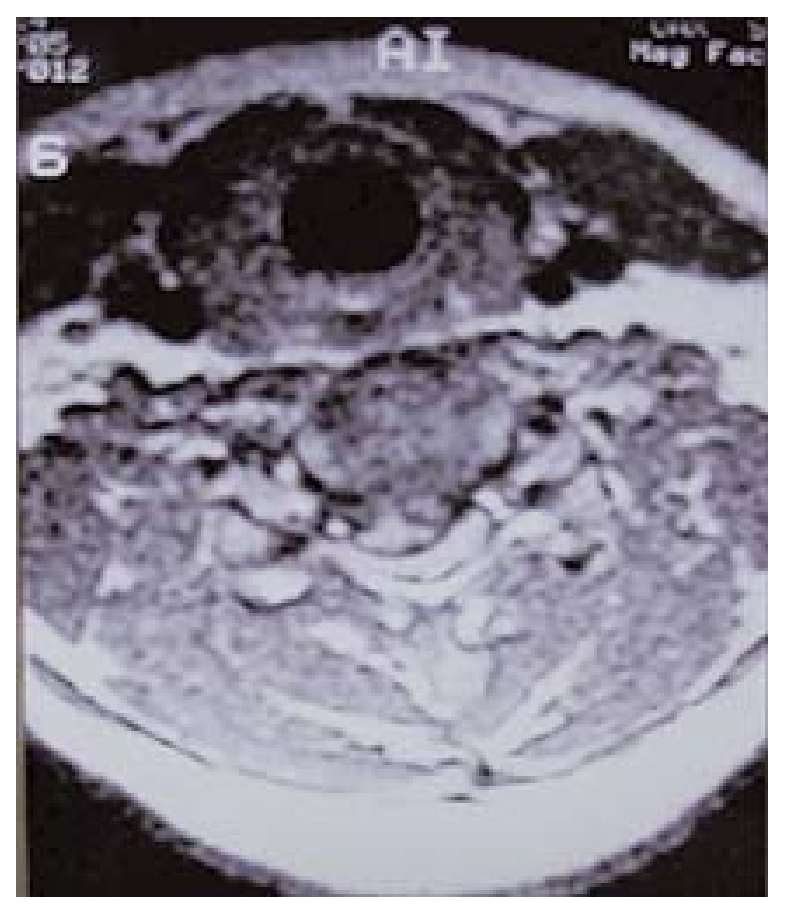

Fig. 2. Case 22. Disc herniation-induced right-sided VA compression at the C3-4 uncovertebral joint. 
who underwent CSMT, chiropractors treated 155, osteopaths nine, osteopaths and chiropractors four, physical therapist one, a patient's spouse one, and unknown practitioners treated two. Of the 172, 32 reported worsening of symptoms after manipulation, but only 21 were found to have irreversible CSMT-related complications associated with manipulation. Of the patients in whom symptoms worsened after CSMT a chiropractor treated 17, an osteopath three, and both a chiropractor and osteopath treated two. Two of the patients in whom incidents occurred were from a different patient population, and these cases were excluded for the purpose of estimating incidence data, but they were included to study the characteristics of this type of injury. Twenty patients were found to have worsened CSMT-induced radiculopathy, 11 suffered worsened myelopathy including two patients with partial Brown-Séquard syndrome, and one patient experienced new onset of vertebrobasilar transient ischemic attacks. The latter patient was found to have a lateral disc herniation compressing the VA. There were no other cases of vascular lesions in the series.

In 20 of 22 patients who underwent surgery, significant improvement was documented in 18; one who did not undergo surgery continues to have symptoms; and one patient was referred for a second-opinion examination only and his outcome is unknown. There were no CSMT-related deaths.

Cases involving the diagnosis of the International Classification Diseases-9 code 722.71 for herniated cervical disc with myelopathy were evaluated between January 1, 1993, and June 30, 1996. During this period, there were 27 patients with cervical disc herniation causing myelopathy and seven in whom symptoms worsened after CSMT. Therefore, in $26 \%$ of all patients surgically treated for cervical disc herniation-induced myelopathy CSMT was the apparent causative factor.

In five patients there was absolute contraindication to manipulation because they suffered neurological deterioration during the course of CSMT, which went unrecognized and manipulation apparently continued. Two patients underwent manipulation of a postsurgical joint, which is a relative contraindication. One patient underwent manipulation despite the presence of radiculopathy, which is a relative contraindication. There was absolute or relative contraindication to CSMT in at least eight of 22 patients. Other contraindications could not be evaluated because it is unknown how many patients underwent premanipultaion imaging studies that might have indicated some contraindication to manipulation.

The number of cervical manipulations before clinical deterioration varied. Deterioration occurred in three patients after the first manipulation, in four after the second manipulation, in 13 after more than two manipulations, and in two patients it could not be determined how many sessions were performed before the final CSMT that caused clinical deterioration.

\section{Estimation of Incidence}

Population estimates for 1995 from the geographical area surrounding Tulsa, Oklahoma, were taken from US Census data. The population of this geographical area was $1,131,638$ in 1995. The estimate of population usage of chiropractic care was found to be $7.5 \%$ during a 3 to 5 -year period. ${ }^{45}$ The range of other estimates of chiropractic care from 45,000 chiropractors in the US was 12 to 125 million visits per year. ${ }^{35,45,46}$ Of these visits, $38 \%$ involved a manipulation on the first visit and 66\% involved manipulation of the spine on subsequent visits. Of these visits, $10.3 \%$ were for facial or neck pain, swelling, or injury and $9.6 \%$ were for complaints of headache. ${ }^{45}$ Based on these data, with our local 20 patients who experienced CSMTrelated complication during the study period, and assuming that 17,000 patients had undergone cervical manipulation during the study period, then the complication rate was approximately one irreversible complication per every 850 patients undergoing a series of manipulations in the local region of the study. If the mean number of manipulations per patient is assumed to be 10 , then the risk of complication in this study would be one in every 8500 cervical manipulations. Using other published data to estimate the number of cervical manipulations in this regional population, there could be up to 180,000 procedures annually, yielding a complication rate of one irreversible per 45,600 cervical manipulations. ${ }^{10}$ This does not take into account that our group represents only one third of the neurosurgeons in the geographical region around Tulsa.

The incidence of complications after CSMT has been estimated in the literature. The most commonly reported complication is vertigo occurring at a rate of 1218 per 1.53 million manipulations, with diminished consciousness occurring in 10 per 1.53 million procedures, loss of consciousness in 12 per 1.53 million procedures, and radicular deficits in 11 per 1.53 million manipulations. ${ }^{13}$ The minimum complication rate for CSMT has been reported at one per 41,500 procedures and the severe complication rate has been reported at a rate of one per $383,750 . .^{13}$ The authors of other studies have noted higher complication rates after CSMT. In one prospective study of 1058 patients, 55\% reported at least one reaction to manipulation: $35 \%$ responses were mild, $50 \%$ moderate, $14 \%$ unpleasant, and $1 \%$ termed unbearable. ${ }^{43}$ The incidence may be higher than expected because, with the population in the region at approximately 1.1 million, only one major complication per year would be expected if every person in the region underwent one cervical manipulation per year.

\section{DISCUSSION}

Cervical spinal manipulation is frequently undertaken in patients with neck pain or headache. The authors of a variety of studies have attested to either the efficacy or lack of efficiency of manipulation therapy. ${ }^{1,6,8,19,22,25,33,34,48}$ Other authors have noted bias in reporting complications of manipulation. ${ }^{50}$ Conservative methods of treating cervical disc disease without manipulation or surgical intervention have been associated with success rates of approximately $80 \% .39,40$ One author wrote that "manipulation of the cervical spine is not to be considered conservative therapy, but rather a most aggressive noninvasive procedure by which the normal mechanical attributes of the motion segments can be restored if aberrant." ${ }^{8}$ In a recent publication the authors did not recommend cervical manipulation because the risk/benefit ratio did not appear to support the practice. ${ }^{35}$ 


\section{Contraindications to Cervical Spine Manipulation}

High-velocity neck manipulation is absolutely contraindicated in patients with rheumatoid arthritis, acute fractures and dislocations, os odontoideum, infection of bone, osseous malignancies, or cervical myelopathy. Cervical spine manipulation is relatively to absolutely contraindicated in patients with benign bone tumors, vertebrobasilar insufficiency, and aneurysm of a major blood vessel. Neck manipulation is relatively contraindicated in cases of joint hypermobility, postsurgical joints, and osteoporosis. ${ }^{51,13}$ Manipulation of the cervical spine in the acute phases of cervical disc herniation in cases associated with deficit is contraindicated until the deficit has resolved. ${ }^{51,13}$ Dvorak, et al. ${ }^{13}$ have written the following: "In the acute phase of cervical disc herniation with neurologic deficit, manipulation and mobilization of the affected segments are contraindicated as there is a high risk of spinal cord compression due to massive prolapse." In cases of traumatic cervical dislocation, manipulation and reduction of the dislocation has in some cases led to neurological deterioration due to disc displacement into the spinal canal, resulting in cord compression. ${ }^{30}$

\section{CONCLUSIONS}

Manipulation of the cervical spine appears to be associated with the possibility of worsening cervical radiculopathy or myelopathy and causing cervical disc herniation; cervical disc herniation can occur in a lateral position, compressing the VA and leading to posterior circulation ischemia. All of these complications have been reported previously except herniated disc-induced compression of the VA.$^{13,27,32}$ The incidence of significant complications appears to be higher in the population studied than has been previously reported. These complications could be lessened by rigorous adherence to published exclusion criteria for manipulation. ${ }^{51}$

\section{References}

1. Anderson R, Meeker WC, Wirick BE, et al: A meta-analysis of clinical trials of spinal manipulation. J Manipulative Physiol Ther 15:181-194, 1992

2. Assendelft WJ, Bouter LM, Knipschild PG: Complications of spinal manipulation: a comprehensive review of the literature. J Fam Pract 42:475-480, 1996

3. Beatty RA: Dissecting hematoma of the internal carotid artery following chiropractic cervical manipulation. J Trauma 17: 248-249, 1977

4. Beneliyahu DJ: Chiropractic management and manipulative therapy for MRI documented cervical disk herniation. J Manipulative Physiol Ther 17:177-185, 1994

5. Blaine ES: Manipulative (chiropractic) dislocations of the atlas. JAMA 85:1356-1359, 1925

6. Brouillette DL, Gurske DT: Chiropractic treatment of cervical radiculopathy caused by a herniated cervical disc. J Manipulative Physiol Ther 17:119-123, 1994

7. Brownson RJ, Zollinger WK, Madiera T, et al: Sudden sensorineural hearing loss following manipulation of the cervical spine. Laryngoscope 96:166-170, 1986

8. Carrick FR: Cervical radiculopathy: the diagnosis and treatment of pathomechanics in the cervical spine. J Manipulative Pysiol Ther 6:129-137, 1983

9. Cortazzo JM, Tom KB: Vertebral artery dissection following chiropractic manipulation. Am J Emerg Med 16:619-620, 1998

10. Dabbs V, Lauretti WJ: A risk assessment of cervical manipulation vs. NSAIDs for the treatment of neck pain. J Manipulative Pysiol Ther 18:530-536, 1995

11. Destee A, Lesoin F, Di Paola F, et al: Intradural herniated cervical disc associated with chiropractic spinal manipulation. J Neurol Neurosurg Psychiatry 52:1113, 1989

12. Donzis PB, Factor JS: Visual field loss resulting from cervical chiropractic manipulation. Am J Ophthalmol 123:851-852, 1997

13. Dvorak J, Kranzlin P, Muhlemann D, et al: Musculoskelatal complications, in Haldeman S (ed): Principles and Practice of Chiropractic. Norwalk CT: Appleton and Lange, 1992, pp 549-578

14. Gallinaro P, Cartesegna M: Three cases of lumbar disc rupture and one of cauda equina associated with spinal manipulation (chiropraxis). Lancet 1:411, 1983 (Letter)

15. Greenman PE: Principles of Manual Medicine, ed 2. Baltimore: Williams and Wilkins, 1996, pp 99-103

16. Heffner JE: Diaphragmatic paralysis following chiropractic manipulation of the cervical spine. Arch Intern Med 145: 562-563, 1985

17. Horn SW Jr: The "locked-in" syndrome following chiropractic manipulation of the cervical spine. Ann Emerg Med 12: 648-650, 1983

18. Hosek RS, Schram SB, Silverman H, et al: Cervical manipulation. JAMA 245:922, 1982

19. Howe DH, Newcombe RG, Wade MT: Manipulation of the cervical spine-a pilot study. J R Coll Gen Pract 33:574-579, 1983

20. Hughes BL: Management of cervical disk syndrome utilizing manipulation under anesthesia. J Manipulative Pysiol Ther 16:174-181, 1993

21. Hurwitz EL, Aker PD, Adams AH, et al: Manipulation and mobilization of the cervical spine. A systematic review of the literature. Spine 21:1746-1760, 1996

22. Jordan A, Bendix T, Nielsen H, et al: Intensive training, physiotherapy, or manipulation for patients with chronic neck pain. A prospective, single-blinded, randomized clinical trial. Spine 23:311-319, 1998

23. Kewalramani LS, Kewalramani DL, Krebs M, et al: Myelopathy following cervical spine manipulation. Am J Phys Med 61:165-175, 1982

24. Klougart N, Leboeuf-Yde C, Rasmussen LR: Safety in chiropractic practice, Part I; the occurrence of cerebrovascular accidents after manipulation to the neck in Denmark from 1978-1988. J Manipulative Physiol Ther 19:371-377, 1996

25. Koes BW, Bouter LM, van Mameren H, et al: The effectiveness of manual therapy, physiotherapy, and treatment by the general practitioner for nonspecific back and neck complaints. A randomized clinical trial. Spine 17:28-35, 1992

26. Krueger BR, Okazaki H: Vertebral-basilar distribution infarction following chiropractic cervical manipulation. Mayo Clin Proc 55:322-332, 1980

27. Lee KP, Carlini WG, McCormick GF, et al: Neurologic complications following chiropractic manipulation: a survey of California neurologists. Neurology 45:1213-1215, 1995

28. Livingston MC: Spinal manipulation causing injury. A three year study. Clin Orthop 81:82-86, 1971

29. Lyness SS, Wagman AD: Neurological deficit following cervical manipulation. Surg Neurol 2:121-124, 1974

30. Mahale YJ, Silver JR, Henderson NJ: Neurological complications of the reduction of cervical spine dislocations. J Bone Joint Surg (Br) 75:403-409, 1993

31. Mas JL, Henin D, Bousser MG, et al: Dissecting aneurysm of the vertebral artery and cervical manipulation: a case report with autopsy. Neurology 39:512-515, 1989

32. Padua L, Padua R, LoMonaco M, et al: Radiculomedullary 
complications of cervical spinal manipulation. Spinal Cord 34: 488-492, 1996

33. Polkinghorn BS: Treatment of cervical disc protrusions via instrumental chiropractic adjustment. J Manipulative Pysiol Ther 21:114-121, 1998

34. Pollard H, Tuchin P: Cervical radiculopathy: a case for ancillary therapies? J Manipulative Pysiol Ther 18:244-249, 1995

35. Powell FC, Hanigan WC, Olivero WC: A risk/benefit analysis of spinal manipulation therapy for relief of lumbar or cervical pain. Neurosurgery 33:73-79, 1993

36. Pratt-Thomas HR, Berger KE: Cerebellar and spinal injuries after chiropractic manipulation. JAMA 133:600-603, 1947

37. Putnam TD, Wu Y: Tracheal rupture following cervical manipulation: late complication posttracheostomy. Arch Phys Med Rehabil 67:48-50, 1986

38. Raskind R, North CM: Vertebral artery injuries following chiropractic cervical spine manipulation-case reports. Angiology 41:445-452, 1990

39. Rothman RH, Marvel JP Jr, Baker R: The conservative treatment of cervical disc disease. Orthop Clin North Am 2: 435-441, 1971

40. Saal JS, Saal JA, Yurth EF: Nonoperataive management of herniated cervical intervertebral disc with radiculopathy. Spine 21: 1877-1883, 1996

41. Schellhas KP, Latchaw RE, Wendling LR, et al: Vertebrobasilar injuries following cervical manipulation. JAMA 244: 1450-1453, 1980

42. Segal DH, Lidov MW, Camins MB: Cervical epidural hematoma after chiropractic manipulation in a healthy young woman: case report. Neurosurgery 39:1043-1045, 1996

43. Senstad O, Leboeuf-Yde C, Borchgrevink C: Frequency and characteristics of side effects of spinal manipulative therapy. Spine 22:435-440, 1997

44. Shafrir Y, Kaufman BA: Quadriplegia after chiropractic manipulation in an infant with congenital torticollis caused by a spinal cord astrocytoma. J Pediatr 120:266-269, 1992

45. Shekelle PG, Brook RH: A community-based study of the use of chiropractic services. Am J Public Health 81:439-442, 1991

46. Shekelle PG, Coulter I: Cervical spine manipulation: summary report of a systematic review of the literature and a multidisciplinary expert panel. J Spinal Disord 10:223-228, 1997

47. Sinel M, Smith D: Thalamic infarction secondary to cervical manipulation. Arch Phys Med Rehabil 74:543-546, 1993

48. Sloop PR, Smith DS, Goldenberg E, et al: Manipulation for chronic neck pain. A double-blind controlled study. Spine 7: 532-535, 1982

49. Smith RA, Estridge MN: Neurologic complications of head and neck manipulations: report of two cases. JAMA 182:528-531, 1962

50. Terrett AG: Misuse of the literature by medical authors in discussing spinal manipulative therapy injury. J Manipulative Pysiol Ther 18:203-210, 1995

51. The Texas Chiropractic Association: Texas Guidelines for Chiropractic Quality Assurance and Practice Parameters. Gaithersburg, MD: Aspen Publishers, 1994, pp 167-177

Manuscript received October 20, 2002.

Accepted in final form November 20, 2002.

Address reprint requests to: David G. Malone, M.D., 1919 South Wheeling, Suite \#600, Tulsa, Oklahoma 74104. email: xmalone @ cox.net. 